\title{
Hypothyroidism and chronic kidney disease: An undervalued two-way relationship
}

Joana Coutinho, Catarina Reis Santos, Ernesto Rocha

Nephrology department, ULS Castelo Branco

\section{ABSTRACT}

There is long-standing evidence that thyroid disease and kidney disease have a two-way relationship, as they can aggravate and lead to each other. The exact nature of this association and its clinical implications have been a matter of debate, as co-sharing of risk factors and appropriate prospective studies that allow for causal relationships to be inferred are lacking.

Hypothyroidism is currently regarded by the American Heart Association as a modifiable cardiovascular risk factor and a reversible cause of heart failure. Among the nephrology community, while hypothyroidism has been an increasingly prevalent issue, formal guidelines on how to proceed when such association occurs are lacking and its clinical implications are often underappreciated among practitioners. Whether renal disease caused the thyroid disturbance or vice-versa, there is evidence pointing to thyroid dysfunction being a risk factor for incident chronic kidney disease and its progression and it is linked to increased overall mortality in CKD patients. The authors provide a review of current scientific evidence on this complex relationship.

Keywords: Cardiovascular risk, Chronic Kidney Disease, Hypothyroidism, Mortality

\section{INTRODUCTION}

Thyroid hormones are critical regulators of cell biology processes such as growth, differentiation and energy production. The kidney, on the other hand, is responsible for the body's internal homeostasis, regulating extracellular water and electrolytes. Though their target of action is different, one at the cellular level and the other at the extracellular, these two organs have long been recognized as working in tandem during pathophysiological processes to maintain cellular, tissue and bodily homeostasis ${ }^{1}$.

The nature of this thyroid-kidney relationship is not yet completely clarified. A significant number of studies into the association between these two diseases have reached conflicting results ${ }^{2}$. One of the difficulties in establishing a causal relationship is the co-sharing of risk factors that may pose as confounders (old age, obesity, smoking, coronary artery disease, hypertension, hyperuricemia). Hypothyroidism is currently regarded as a modifiable cardiovascular risk factor by the American Heart Association (AHA $)^{3}$ and an increasingly recognized risk factor for CKD progression ${ }^{2}$. Hypothyroidism is linked to worse outcomes in CKD patients ${ }^{4,5}$, which are possibly explained by additional mechanisms other than the traditional cardiovascular system. The authors provide a review of current scientific evidence on this topic.

\section{METHODS}

Literature search was performed using Pubmed and Cochrane Library databases and the search terms "hypothyroidism and chronic kidney disease" and with a combination of "cardiovascular disease", "vascular calcification", "thyroid hormonal replacement", "dyslipidemia", "anemia", "cognitive dysfunction" with "thyroid disorders" and "kidney disease". We identified further references from the original articles and studied only English language articles. Priority was given to articles reporting prospective studies and randomized controlled studies.

\section{DISCUSSION}

\section{Mechanisms by which thyroid disturbances influence renal disease}

Thyroid hormones exert effects on the kidney both at the structural and functional level, influencing its glomerular, tubular and endocrine functions ${ }^{6}$. Such effects are very much dependent on age and human development stage ${ }^{7}$. T3 and T4 are biological activators of kidney growth and development, critical to the fetal and early infancy period. In fact, an increased prevalence of renal and urinary tract anomalies has been observed in children with congenital hypothyroidism ${ }^{8}$. After the early infancy period, thyroid hormones exert direct effects on physico-chemical functions of the kidney, affecting its tubular and endocrine functions, and affect the kidney indirectly through their influence on cardiac and endothelial function.

Primary hypothyroidism can be linked to an increase in serum creatinine due to reduction of glomerular filtration rate in more than a half of patients ${ }^{9}$. This elevation of serum creatinine is reversible with hormonal substitution ${ }^{10}$. Hypothyroid state influence on 
glomerular function was historically attributed to its influence on the cardiac-endothelial system with decreasing glomerular filtration and renal plasma flow resulting from reduced cardiac output, increased systemic vascular resistance and decreased arterial compliance [1114]. Over the last few decades, thyroid hormones' influence on other hemodynamic factors, namely the renin-angiotensin-aldosterone (RAS) system, and metabolic factors has been increasingly recognized. Hypothyroidism is now regarded as an important cardiovascular risk factor leading to cardiac dysfunction, atherosclerosis and hyperlipidemia, which may explain why hypothyroidism accelerates CKD progression $^{15}$

Cardiac function is directly affected by thyroid hormones through genomic and non-genomic mechanisms. T3, the biologically active form, is responsible for the regulation of gene expression of structural proteins crucial for myocardial contractility/ relaxation system, cardiac conduction system, and through non genomic pathways, T3 modulates myocardial oxygen consumption ${ }^{11}$. Overt hypothyroidism is clearly linked to left ventricular diastolic dysfunction and T3 levels have been shown to correlate well with heart failure functional class as classified by the New York Heart Association (NYHA) [1618]. Cardiac dysfunction in hypothyroidism is indirectly aggravated by reduced peripheral oxygen consumption which reduces the release of vasoactive agents by the endothelium and promotes impaired vasorreactivity and arterial stiffness ${ }^{19}$. The metabolic disturbances associated with hypothyroid states will further contribute to a pro-atherogenic state, which possibly impacts on CKD progression. Hypothyroidism is associated with hyperlipidemia and atherosclerosis, namely increased LDL and decreased Apolipoprotein A, mainly due to reduced activity of the hepatic LDL receptor and hepatic lipoprotein lipase $\mathrm{e}^{20-24}$.

Thyroid hormones can influence RAS system directly or by increasing the response of tissues to the action of the sympathetic system; however the intricate mechanistic pathways remain to be elucidated ${ }^{24-27}$. Thyroid hormones' effects on RAS components are complex and dependent on age ${ }^{25-27}$. Thyroid hormones modulate renin gene expression and angiotensin converting enzyme (ACE) activity at both circulating and tissue levels ${ }^{28}$. The reduction of ACE activity in the kidney of rat models with overt hypothyroidism was reversible by hormonal replacement ${ }^{29}$ as was plasma renin activity reduction. However, only one study analyzed RAS activity in subclinical thyroid disorders, finding no differences in plasma renin activity or plasma aldosterone between patients with subclinical hypothyroidism and normal individuals ${ }^{30}$.

Thyroid hormones' influence on tubular functions of the kidney occurs mainly through modulation of ATPase pump gene transcription, reducing its activity and interfering with tubular reabsorption of sodium and calcium. Hyponatremia is a common complication of overt hypothyroidism and is mostly attributed to impaired water excretion by thyroid hormones influence on the NHE3 transporter and NaPi2 transporter. Increased distal delivery of sodium is the leading cause of hypothyroidism urinary concentrating deficit, as expression and activity of other transporters such as NKCC2 and AQP2 have proven to be unchanged in hypothyroidism ${ }^{31}$. Urinary calcium excretion parallels to sodium excretion in thyroid deficiency; however it is rarely clinically significant ${ }^{32}$.

\section{Aging, thyroid hormones and vascular calcification: a very complex system}

Over the last decade, efforts have been made to investigate thyroid hormone influence in medial vascular calcification, a nontraditional cardiovascular risk factor of CKD patients. In prevalent peritoneal dialysis patients, while T3 levels correlate inversely to coronary artery scores and arterial stiffness ${ }^{33}$, the intervening factors on the molecular level and their part in such a complex system is intriguing. Klotho and matrix GLA protein (MGP), known participants in the CKD-vascularbone system, are so far the main culprits in thyroid-related vascular calcification ${ }^{34}$. At present, evidence points to the fact that CKD and hypothyroidism could synergistically promote vascular calcification.

Klotho is a hormone produced by the distal nephron that can be circulating/soluble or membrane-bound, with different clinical implications at each level. Klotho deficiency is known to occur early in CKD ${ }^{35}$. Membrane-bound Klotho functions as co-receptor for Fibroblast Growth Factor-23 (FGF23) and Klotho deficiency in CKD could cause resistance to FGF23 action on the kidney as a phosphaturic agent ${ }^{35,36}$. The hyperphosphatemia would then promote vascular smooth muscle cells' osteogenic differentiation ${ }^{37}$. Matrix Gla Protein gene expression, a natural inhibitor of such osteogenic differentiation, seems regulated by thyroid hormones, and is decreased in vitro in hypothyroidism ${ }^{34}$.

Soluble Klotho is the only known mammalian anti-aging hormone and its deficiency is associated to organ atrophy, infertility, vascular calcification, atherosclerosis, osteomalacia, osteoporosis, peripheral insulin sensitivity, metabolic disturbances, and cerebral changes, all of which occur in "normal" aging ${ }^{38}$. Klotho synthesis occurs in the distal nephron under control of thyroid hormone, and Klotho deficiency should lead to accelerated aging; however, thyroid influence at the tissue level is dependent on the developmental stage. In fact, evidence from observational studies suggest there is an age-dependent susceptibility to mild hypothyroidism and in the extreme elderly (>85 years of age) this may actually confer a protective mechanism for protein catabolism and energy saving, reducing their cardiovascular risk ${ }^{39,40}$. In younger end stage renal disease patients, however, free T3 levels were positively associated with circulating levels of MGP and Klotho and correlated to vascular calcification scores, suggesting there might be a synergism ${ }^{34}$.

\section{Other complications of CKD in which hypothyroidism may play a role}

Thyroid hormones are involved in the regulation of erythroid differentiation and modulate erythropoietin (EPO) gene expression. It has been suggested that in hypothyroid conditions, reduced EPO levels might account for anemia ${ }^{41}$. However, thyroid hormones also influence peripheral tissue oxygen consumption and seem to influence other participants in erythropoiesis, such as hypoxia inducible factor 1 , which is known to induce various forms of anemia. Overt hypothyroidism was proposed as a cause of EPO resistance in dialysis patients, assuming euthyroid status is needed for a normal response to recombinant $\mathrm{EPO}^{42}$; however only one observational study has established the relationship between hypothyroidism and EPO dosages ${ }^{43}$. Further studies are warranted to evaluate whether thyroid hormone 
replacement reduces EPO and iron needs in CKD patients. Meanwhile, hypothyroidism should be regarded as a contributing factor to anemia in CKD patients.

Mild cognitive dysfunction is a recently recognized complication of CKD ${ }^{44}$. According to the U.S. Renal Data System, it more than doubles the mortality risk of CKD patients and increases days spent in hospital $^{45}$. Hypothyroidism could both contribute to the vascular calcification that is thought to underlie the white matter degeneration that is seen in $C K D^{46}$, or could act synergistically, further compromising specific cognitive domains of memory and executive function, as is reported in hypothyroid disorders ${ }^{47,48}$. Though classically these two conditions cause a different spectrum of neurologic disorders, symptoms may overlap and hypothyroidism is a condition that must be excluded when considering the mild cognitive impairment in CKD patients.

\section{Mechanisms by which kidney disease influences thyroid function}

The kidney is responsible for degradation and excretion of thyroid hormones and iodine clearance, so understandably kidney dysfunction will interfere with thyroid hormone levels ${ }^{15}$. As thyroid hormones are protein-bound, kidney diseases associated to proteinuria and/or increased protein catabolism will also indirectly influence thyroid hormone bioavailability. Theoretically speaking, one could divide thyroid hormone abnormalities into those induced by the uremic milieu of renal disease and those related to renal substitution therapy.

Thyroid stimulating hormone (TSH) is cleared by the kidneys and increased half-life of TSH is the norm in CKD; however even at lower glomerular filtration rates, TSH levels seem to remain normal in the majority of patients ${ }^{15}$. The increased half-life of TSH seems to blunt TSH response to Thyroid releasing hormone (TRH). Reduction in glomerular filtration rate leads to reduced iodine clearance, contributing to enlargement and colloid degeneration of the of the thyroid gland. Thyroid gland colloid degeneration is generally insidious, with very subtle clinical changes and not clinically relevant for most patients. Nevertheless, dialysis has been associated to an increased risk of thyroid nodules and thyroid cancer ${ }^{49}$.

On the other hand, the uremic milieu can blunt thyroid hormones' bioavailability to peripheral tissues, leading to a state of thyroid resistance. T3 and T4 level abnormalities are the most common in CKD and low T3 levels is by far the most common abnormality observed in advanced CKD stages ${ }^{50}$; however this may just reflect reduced availability to peripheral tissues due to low protein status or reduced peripheral conversion of T4 to its active form, T3. Factors that contribute to this low activity of iodothyronine deiodinase are malnutrition, inflammation and metabolic acidosis, all of which are fairly prevalent in CKD.

\section{Figure 1}

Pathophysiological changes of thyroid hormones in chronic kidney disease. TSH - thyroid stimulating hormone. TRH - thyroid releasing hormone.

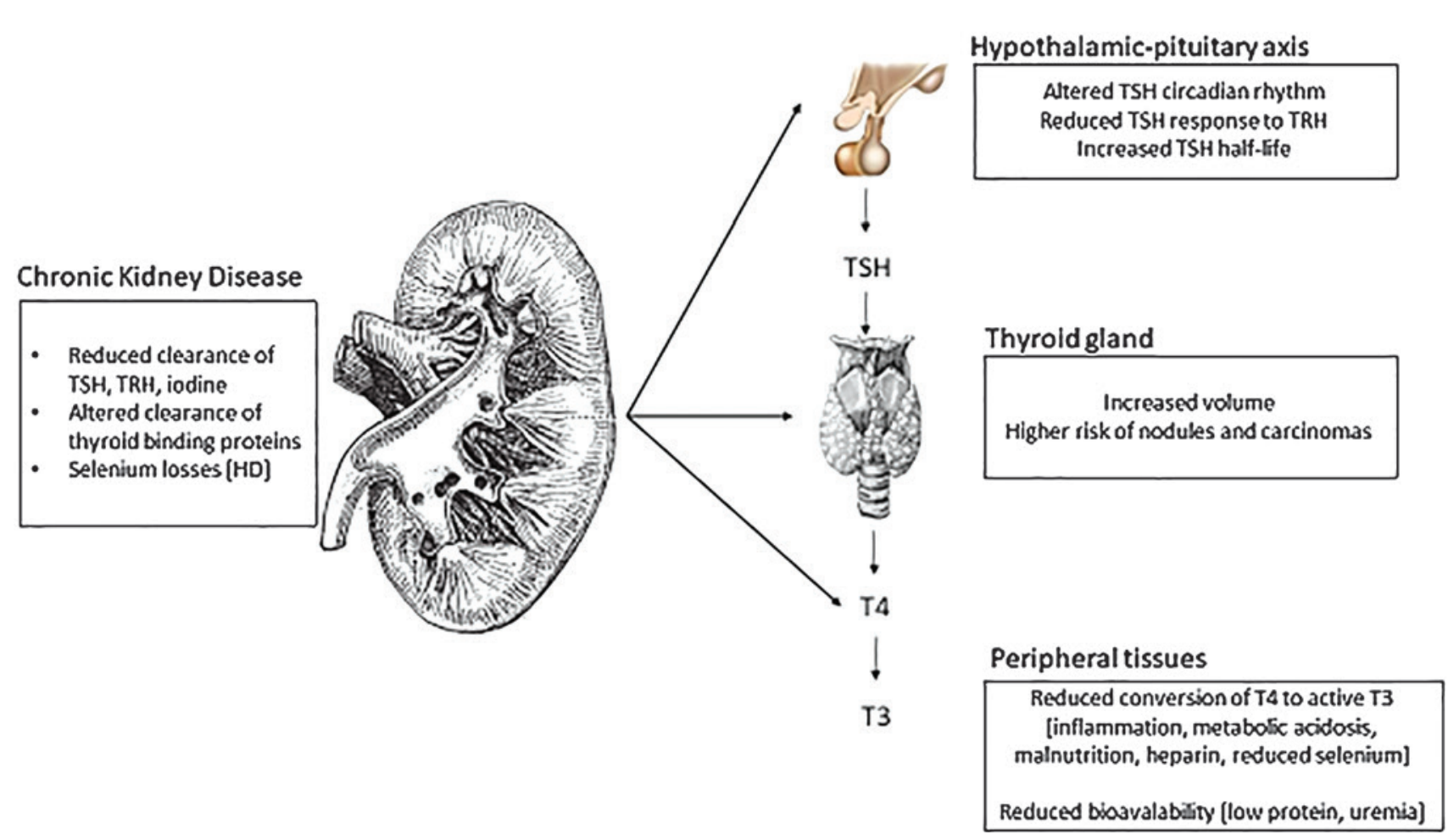


In the third National Health and Nutrition Examination Survey (NHANES III), hypothyroidism was reported to be increasingly prevalent as glomerular filtration rate declines, affecting $23 \%$ in patients CKD stage 5 , with more than half of the patients having subclinical hypothyroidism ${ }^{51,52}$. In the few studies conducted in the dialysis population, the prevalence of subclinical hypothyroidism has been the greatest in peritoneal dialysis patients, presumably due to higher protein losse $^{53}$. In the hemodialysis population, additional factors contributing to thyroid hormones disturbances are the use of heparin, which reduces T4 bioavailability to peripheral tissues, and greater losses of selenium, a trace metal that modulates peripheral conversion of T4 to $T 3^{54}$. Despite the initial enthusiasm regarding selenium supplementation to correct for thyroid hormones disturbances, this has not been proven useful in clinical practice ${ }^{55,56}$

A summary of the pathophysiological changes in thyroid hormones observed in CKD is provided schematically in Figure 1.

\section{Clinical implications of thyroid disease for CKD patients}

The American Heart Association guidelines in 2016 included overt hypothyroidism as a reversible cause of heart failure, making formal recommendations for treatment with hormonal replacement therapy, which can improve cardiac function ${ }^{3}$. In subclinical hypothyroidism, while some of the same biological disturbances have been observed, namely increased systemic vascular resistance, arterial stiffness, and altered endothelial function ${ }^{22}$, there is no consensus on whether hormonal replacement can be of benefit to stabilize heart function and reduce cardiovascular mortality 57,58 .

In CKD patients, hypothyroidism, even subclinical, is linked to accelerated GFR decline and increased mortality ${ }^{5,59-62}$. Hormonal replacement therapy can potentially reduce GFR decline ${ }^{63,64}$. A study by Shin et al into patients with CKD stage 2 to 4 with subclinical hypothyroidism demonstrated that the rate of decline in eGFR was significantly attenuated by levothyroxine therapy to TSH within normal range target $\left(-4.31 \pm 0.51\right.$ vs. $-1.08 \pm 0.36[\mathrm{~mL} / \mathrm{min}] /\left[\right.$ year $\left.\left.\cdot 1.73 \mathrm{~m}^{2}\right], p<0.001\right)$, even after adjustment for age, sex, diabetes, mean arterial pressure, and serum albumin, cholesterol, and triglyceride concentrations $(p<0.001)^{65}$.

Lastly and most importantly, thyroid disease in CKD patients has been clearly linked to an increase in overall mortality, not just in endstage renal failure, but also in earlier stages of CKD, as recently recognized in a study by Rhee et al $2018^{5}$. In Rhee's cohort of patients with CKD stage 3, there was an increased risk of death from $17 \%$ to $27 \%$ for hypothyroid patients, over a median follow-up period of 5.5 years.

\section{FINAL REMARKS AND TAKE HOME MESSAGES}

In the light of current evidence, thyroid functional disease must be considered as much a modifiable cardiovascular risk factor for CKD patients, as it is for heart failure patients, if not a risk factor for CKD progression itself. Apart from contributing to cardiovascular risk, there is growing evidence that hypothyroidism may impact other CKD complications, such as vascular calcification, anemia or mild cognitive impairment. Hypothyroidism is certainly associated to mortality across different CKD stages. As such, hypothyroid CKD patients, regardless of CKD stage, should be treated to target normal TSH levels, as it is probably the most reliable surrogate measure of thyroid function. Exception should probably be made for asymptomatic extreme elderly patients (>85 years of age) in whom hypothyroidism has been hypothesized to be a protective mechanism for protein catabolism and energy saving.

Subclinical hypothyroidism is the most conflicting issue, as studies suggest it may have prognostic value in a specific population, namely CKD patients. Shin et al. published very interesting data on how CKD patients with subclinical hypothyroidism should be treated with hormonal replacement, as an improvement of cardiovascular function could stabilize progression of CKD. Most studies into the treatment of subclinical hypothyroidism have excluded patients aged $<18$ years or $>75$ years, pregnant, with terminal malignancy or nephrotic range proteinuria. Taking these considerations into account, the authors suggest that CKD patients who should be considered for treatment are those with CKD stages 2 to 5, including CKD5D, aged 18 to 75 years, not pregnant, not nephrotic and without malignancy, who have unspecific symptoms of hypothyroidism (such as depression, fatigue or constipation), high titers of anti-TPO and dyslipidemia. Treatment should be initiated with the lowest dose of levothyroxine available, $0.25 \mathrm{ug}$, as the toxic-to-therapeutic window of levothyroxine treatment is narrow. After treatment initiation, TSH levels should be monitored after 6 weeks and adjustments made to target TSH within normal reference range levels. After achieving target TSH, levels should be monitored every 3 months.

Clearly, the thyroid-kidney interaction cannot be disregarded and nephrologists need to be increasingly aware of hypothyroidism as a risk factor for cardiovascular disease. However, further studies are needed to understand the exact mechanistic pathways of this interaction and who is likely to benefit from hormonal replacement therapy. A well-designed prospective double-blind randomized trial (THYROIDHD) has just started recruitment to investigate whether levothyroxine therapy improves outcomes in hemodialysis patients with subclinical hypothyroidism ${ }^{66}$, which is a first step to providing advancements in this field.

\section{Disclosure of potential conflicts of interest: none declared}

\section{References}

1. Mohamedali M, Maddika SR, Vyas A, Iyer V, Cheriyath P. Thyroid disorders and chronic kidney disease. Int J Nephrol 2014; 2014: 520281.

2. Rhee CM, Brent GA, KOvesdy CP, et al. Nephrol Dial Transplant 2014; 0:1-15.

3. Ponikowski P, Voors AA, Anker SD. 2016 ESC Guidelines for the diagnosis and treatment of acute and chronic heart failure. Eur Heart J 2016; 37(27): 2129-2200.

4. Rhee CM, Alexander EK, Bhan I, Brunell SM. Hypothyroidism and mortality among dialysis patients. Clin J Am Soc Nephrol 2013; 8: 593-601.

5. Rhee CM, Kalantar-Zadeh K, Ravel V, et al. Thyroid status and death risk in US veterans with chronic kidney disease. Mayo Clin Proc 2018; 93(5): 573-585.

6. Kaptein EM. Thyroid hormone metabolism and thyroid diseases in chronic renal failure. Endocr Rev 1996; 17(1): 45-63.

7. Iglesias P, Díez JJ. Thyroid dysfunction and kidney disease. Eur J Endocrinology 2009; 160: 503-515.

8. Kumar J, Gordillo R, Kaskel FJ, Druschel CM, Woroniecki RP. Increased prevalence of renal and urinary tract anomalies in children with congenital hypothyroidism. J. Pediatr. 2009; 154: 263-266.

9. Montenegro J, González O, Saracho R, Aguirre R, Martínez I. Changes in renal function in primary hypothyroidism. Am J Kidney Dis 1996; 27: 195-198. 
10. Kreissman SH, Hennessey JV. Consistent reversible elevations in serum creatinine levels in severe hypothyroidism. Arch Intern Med 1999; 159: 79-82.

11. Klein I, Ojamaa K. Thyroid hormone and the cardiovascular system. New Eng J Med 2001; 344: 501-509.

12. Klein I, Danzi S. Thyroid disease and the heart. Circulation 2007; 116: 1725-1735.

13. Crowley WF, Ridgway EC, Bough EW, Francis GS, Damiels GH. Non invasive evaluation of cardiac function in hypothyroidism. Response to gradual thyroxine replacement. New Eng J Med 1977; 29: 1-6.

14. Tang YD, Kuzman JA, Said S, Anderson BE, Wang X, Gerdes AM. Low thyroid function leads to cardiac atrophy with chamber dilatation, impaired myocardial blood flow, loss of arterioles, and severe systolic dysfunction. Circulation 2005; 112: 3122-3130.

15. Rhee CM. Interaction between thyroid and kidney disease: An overview of evidence. Curr Opin Endocrinol Diabetes Obes 2016 Oct; 23(5): 407-415.

16. Gerdes MA, Lervasi $G$. Thyroid replacement therapy and heart failure. Circulation 2010; 122:385393.

17. Aghini-Lombardi F, Di Bello V, Talini E, et al. Early textural and functional alterations of left ventricular myocardium in mild hypothyroidism. Eur J Endocrinol 2006; 155: 3-9.

18. Yazici M, Gorgulu S, Sertbas $Y$, et al. Effects of thyroxin therapy on cardiac function in patients with subclinical hypothyroidism: Index of myocardial performance in the evaluation of left ventricular function. Int J Cardiol 2004; 95: 135-143.

19. Danzi S, Klein I. Thyroid hormone and the cardiovascular system. Minerva Endocrinol 2004;29(3):139-150.

20. Duntas LH. Thyroid disease and lipids. Thyroid 2002; 12: 287-293.

21. Cappola AR, Ladenson PW. Hypothyroidism and atherosclerosis. J Clin Endocrinol Metab 2003; 88: 2438-2444.

22. Biondi B, Cooper DS. The clinical significance of subclinical thyroid dysfunction. Endocr Rev 2008; 29(1): 76-131.

23. Kahaly GJ. Cardiovascular and atherogenic aspects of subclinical hypothyroidism. Thyroid 2000; 10: 665-679.

24. Kobori H, Ichihara A, Suzuki H, Miyashita Y, Hayashi M, Saruta T. Thyroid hormone stimulates renin synthesis in rats without involving the sympathetic nervous system. Am J Physiol Cell Physiol 1997; 272: 227-232.

25. Vargas F, Rodríguez-Gómez I, Vargas-Tendero $P$, Jimenez $E$, Montiel $M$. The renin-angiotensin system in thyroid disorders and its role in cardiovascular and renal manifestations. J Endocrinol 2012; 213: 25-36.

26. Kobori $H$, Hayashi M, Saruta T. Thyroid hormone stimulates renin gene expression through the thyroid hormone response element. Hypertension 2001; 37: 99-104.

27. Jiménez E, Montiel M, Narváez JA, Morell M. Renin-angiotensin system in hypothyroid rats: effects of potassium iodide and triiodo-L-thyronine. Acta Endocrinologica 1984; 105: 505-510.

28. Montiel $M$, Ruiz $M$, Jiménez $E$, Morell $M$. Angiotensin converting enzyme in hyper- and hypothyroid rats. HORM METAB RES 1987; 19: 90-92.

29. Carneiro-Ramos MS, Diniz GP, Almeida J, et al. Cardiac angiotensin II type I and type I receptors are increased in rats submitted to experimental hypothyroidism. J. Physiol 2007; 583: 213-223

30. Villabona C, Sahun M, Roca M, et al. Blood volumes and renal funcion in overt and subclinical primary hypothyroidism. Am J of Medical Sciences 1999; 318: 277-280.

31. Schmitt R, Klussmann E, Kahl T, Ellison DH, Bachmann S. Renal expression of sodium transporters and aquaporin-2 in hypothyroid rats. Am J Physiol Renal Physiol 2003; 284: F1097-F1104.

32. McCaffrey C, Quamme GA. Effects of thyroid status on renal calcium and magnesium handling. Can J Comp Med 1984; 48(1): 51-57.

33. Meuwese CL, Carrero JJ, Cabezas-Rodriguez I, et al. Non-thyroidal illness: A risk factor for coronary calcification and arterial stiffness in patients undergoing peritoneal dialysis. J Intern Med 2013;274(6): 584-593.

34. Meuwese $\mathrm{CL}$, Olauson $\mathrm{H}$, Qureshi AR, et al. Associations between thyroid hormones, calcification inhibitor levels and vascular calcification in end-stage renal disease. PLOS ONE 2015; 10(7): e0132353.

35. Hu MC, Kuro-o M, Moe MW. Renal and extra-renal actions of Klotho. Semin Nephrol 2013; 33: 118-129.

36. Villanueva SL, Sánchez González C, Sánchez Tomero JA, Aguilera A, Ortega Junco E. Enfermedad óseo mineral relacionada con la enfermedad renal crónica: Klotho y FGF23; implicaciones cardiovasculares. Nefrología 2016; 36: 368-375

37. Chen NX, O'Neill KD, Duan D, Moe S. Phosphorus and uremic serum up-regulate osteopontin expression in vascular smooth muscle cells. Kidney Int 2002; 62; 1724-1731.

38. Kuro-o M, Matsumura $Y$, Aizawa $H$, et al. Mutation of the mouse klotho gene leads to a syndrome resembling ageing. Nature 1997; 390: 45-51.

39. Razvi S, Shakoor A, Vanderpump M, Weaver JU, Pearce SH. The influence of age on the relationship between subclinical hypothyroidism and ischemic heart disease: A metaanalysis. $J$ Clin Endocrinol Metab 2008; 93(8): 2998-3007.

40. Mariotti S. Mild hypothyroidism and ischemic heart disease: Is age the answer? J Clin Endocrinol Metab 2008; 93(8): 2969-2671.
41. Maggio M, De Vita F, Fisichella A, et al. The role of the multiple hormonal dysregulation in the onset of "anemia of aging": focus on testosterone, IGF-1, and thyroid hormones. Int J Endocrinol 2015; 2015: 292574.

42. Dilek M, Akpolat T, Cengiz K. Hypothyroidism as a cause of resistance to erythropoietin. Nephron 2002; 92: 248.

43. Ng YY, Lin HD, Wu SC, et al. Impact of thyroid dysfunction on erythropoietin dosage in hemodialysis patients. Thyroid 2013;23(5): 552-561.

44. Viggiano D, Carsten AW, Blankestijn PJ, et al. Mild cognitive impairment and kidney disease: clinical aspects. Nephrol Dial Transplant 2019; 1-8.

45. U.S. Renal Data System. USRDS 2006 Annual Data Report: Atlas of Chronic Kidney Disease \& End Stage Renal Disease in the United States. 2006

46. Lau WL, Huisa BN, Fisher M. The cerebrovascular-chronic kidney disease connection: Perspectives and mechanisms. Transl Stroke Res 2017 Feb; 8(1): 67-76.

47. Constant EL, Adam S, Seron X, Bruyer R, Seghers A, Daumerie C. Anxiety and depression, attention, and executive functions in hypothyroidism. J Int Neuropsychol Soc 2005 Sep; 11(5): 535-44.

48. Samuels MH. Psychiatric and cognitive manifestations of hypothyroidism. Curr Opin Endocrinol Diabetes Obes 2014 Oct; 21(5): 377-383.

49. Wong G, Staplin N, Emberson J, et al. Chronic kidney disease and the risk of cancer: An individual patient data meta-analysis of 32,057 participants from six prospective studies. BMC Cancer 2016; 16: 488.

50. Zoccali C, Mallamaci F, Tripepi G, Cutrupi S, Pizzini P. Low triiodothyronine and survival in end-stage renal disease. Kidney Int 2006; 70: 523-528.

51. Lo JC, Chertow GM, Go AS, Hsu CY. Increased prevalence of subclinical and clinical hypothyroidism in persons with chronic kidney disease. Kidney Int 2005; 67(3): 1047-1052.

52. Chonchol M, Lippi G, Salvagno G, Zoppini G, Muggeo M, Targher G. Prevalence of subclinical hypothyroidism in patients with chronic kidney disease. Clin J Am Soc Nephrol 2008; 3(5): 12961300.

53. Ng YY, Wu SC, Lin HD, et al. Prevalence of clinical and subclinical thyroid disease in a peritoneal dialysis population. Peritoneal dialysis international: journal of the International Society for Peritoneal Dialysis 2012; 32: 864-893.

54. Foote JW, Hinks L, Lloyd B. Reduced plasma and white cell selenium levels in hemodialysis patients. Clin Chim Acta 1987; 164: 323-328.

55. Georges MD, Bonnefont DJ, Bourely BA, et al. Correction of selenium deficiency in hemodialyzed patients. Kidney Int Suppl 1989; 27: 274-277.

56. Omrani HR, Rahimi M, Nikseresht $K$. The effect of selenium supplementation on acute phase reactants and thyroid function tests in hemodialysis patients. Nephrourol Mon 2015 Mar; 7(2):e24781.

57. Hassan A, Altamirano-Ufion A, Zulfigar Bm Boddu P. Sub-clinical hypothyroidism and its association with increased cardiovascular mortality: Call for action. Cardiol Res 2017 Apr; 8(2): 31-35.

58. Hayashi T, Hasegawa T, Kanzaki H, et al. Subclinical hypothyroidism is an independent predictor of adverse cardiovascular outcomes in patients with acute decompensated heart failure. ESC Heart Fail 2016; 3(3): 168-176.

59. Huang X, Ding L, Peng K, et al. Thyroid hormones associate with risk of incident chronic kidney disease and rapid decline in renal function: a prospective investigation. J. Transl. Med. 2016; 14: 336.

60. Chuang M, Liao K, Hung Y, Wang PY, Chou Y, Chou P. Association of TSH elevation with all-cause mortality in elderly patients with chronic kidney disease. PLoS One 2017; 12(1): e0168611.

61. Rhee CM, Kim S, Gillen DL, et al. Association of thyroid functional disease with mortality in a national cohort of incident hemodialysis patients. J Clin Endocrinol Metab 2015; 100: 1386-1395.

62. Drechsler C, Schneider A, Gutjahr-Lengsfeld L, et al. Thyroid function, cardiovascular events, and mortality in diabetic hemodialysis patients. Am J Kidney Dis 2014; 63: 988996.

63. Den Hollander JG, Wulkan RW, Mantel MJ, Berghout A. Correlation between severity of thyroid dysfunction and renal function. Clin Endocrinol (Oxf) 2005; 62(4): 423-427.

64. Shin DH, Lee MJ, Kim SJ, et al. Preservation of renal function by thyroid hormone replacement therapy in chronic kidney disease patients with subclinical hypothyroidism. J Clin Endocrinol Metab 2012; 97(8): 2732-2740.

65. Shin DH, Lee MJ, Lee HS, et al. Thyroid hormone replacement therapy attenuates the decline of renal function in chronic kidney disease patients with subclinical hypothyroidism. Thyroid 2013; 23(6): 654-661.

66. $\mathrm{https://clinicaltrials.gov/show/nct03977207}$

\section{Correspondence to:}

Joana Coutinho, MD

Nephrology department, ULS Castelo Branco

Avenida Pedro Alvares 6000-084 Castelo Branco Portugal

E-mail: jcoutinho@ulscb.min-saude.pt 